\title{
Single-molecule mechanical unfolding and folding of a pseudoknot in human telomerase RNA
}

\author{
GANG CHEN, JIN-DER WEN, and IGNACIO TINOCO JR. \\ Department of Chemistry, University of California at Berkeley, Berkeley, California 94720, USA
}

\begin{abstract}
RNA unfolding and folding reactions in physiological conditions can be facilitated by mechanical force one molecule at a time. By using force-measuring optical tweezers, we studied the mechanical unfolding and folding of a hairpin-type pseudoknot in human telomerase RNA in a near-physiological solution, and at room temperature. Discrete two-state folding transitions of the pseudoknot are seen at $\sim 10$ and $\sim 5$ piconewtons $(\mathrm{pN})$, with ensemble rate constants of $\sim 0.1 \mathrm{sec}^{-1}$, by stepwise force-drop experiments. Folding studies of the isolated $5^{\prime}$-hairpin construct suggested that the $5^{\prime}$-hairpin within the pseudoknot forms first, followed by formation of the $3^{\prime}$-stem. Stepwise formation of the pseudoknot structure at low forces are in contrast with the one-step unfolding at high forces of $\sim 46 \mathrm{pN}$, at an average rate of $\sim 0.05 \mathrm{sec}^{-1}$. In the constant-force folding trajectories at $\sim 10 \mathrm{pN}$ and $\sim 5 \mathrm{pN}$, transient formation of nonnative structures were observed, which is direct experimental evidence that folding of both the hairpin and pseudoknot takes complex pathways. Possible nonnative structures and folding pathways are discussed.
\end{abstract}

Keywords: single-molecule; optical tweezers; telomerase RNA pseudoknot; folding pathways; unfolding/folding kinetics; force-jump/drop

\section{INTRODUCTION}

RNA must adopt various secondary and tertiary structures for its diverse biological functions (Tinoco and Bustamante 1999; Gesteland et al. 2006). The building blocks for secondary structure include canonical stems, hairpins, internal loops, bulges, and multibranches; tertiary structure involves interactions between the secondary structure building blocks, for example, loop-stem, looploop, and stem-stem interactions (Tinoco 1996; Tinoco and Bustamante 1999; Leontis et al. 2006). A hairpin (H)-type pseudoknot (Fig. 1B) involves base-pairing between nucleotides in a hairpin loop with nucleotides in a single-stranded region outside the hairpin (Pleij et al. 1985; Puglisi et al. 1988; Tinoco 1996; Su et al. 1999; Staple and Butcher 2005). The H-type pseudoknot is stabilized by base-pairing interactions in the two stems ( $5^{\prime}$ stem 1 and $3^{\prime}$ stem2), which in turn facilitate the tertiary interactions involving loop-stem and loop-loop contacts. The thermodynamics of the H-type pseudoknot formation has been

Reprint requests to: Ignacio Tinoco Jr., Department of Chemistry, University of California at Berkeley, Berkeley, CA 94720, USA; e-mail: intinoco@lbl.gov; fax: (510) 643-6232.

Article published online ahead of print. Article and publication date are at http://www.rnajournal.org/cgi/doi/10.1261/rna.676707. studied by thermal unfolding (Puglisi et al. 1988; Wyatt et al. 1990; Theimer and Giedroc 2000; Theimer et al. 2003, 2005; Soto et al. 2007), but unfolding/folding dynamics and kinetics information are scarce (Wyatt et al. 1990; Green et al. 2007).

Recent developments in single-molecule techniques have facilitated RNA unfolding and folding reactions in near-physiological solutions and temperatures by applying mechanical force and following the reaction trajectories of individual molecules (Liphardt et al. 2001; Onoa et al. 2003). Mechanical unfolding/folding dynamics, kinetics, and thermodynamics information can provide insight into mechanical processes in biology (Bustamante et al. 2004; Tinoco et al. 2006). Here, we use optical tweezers (Smith et al. 1996; Tinoco et al. 2006) to study the mechanical unfolding and folding of a modified pseudoknot in human telomerase RNA (Fig. 1B; Cech 2004; Chen and Greider 2004; Autexier and Lue 2006; Blackburn 2006; Collins 2006; Legassie and Jarstfer 2006; Theimer and Feigon 2006), one molecule at a time, at 200 $\mathrm{mM} \mathrm{NaCl}, 10 \mathrm{mM}$ Tris- $\mathrm{HCl}$ (pH 7.3), $0.1 \mathrm{mM}$ EDTA, at room temperature. This H-type pseudoknot was chosen as a model system because there are three-dimensional NMR structures available for a truncated pseudoknot and a constituent 5'-hairpin (Fig. 1E; Theimer et al. 2003, 


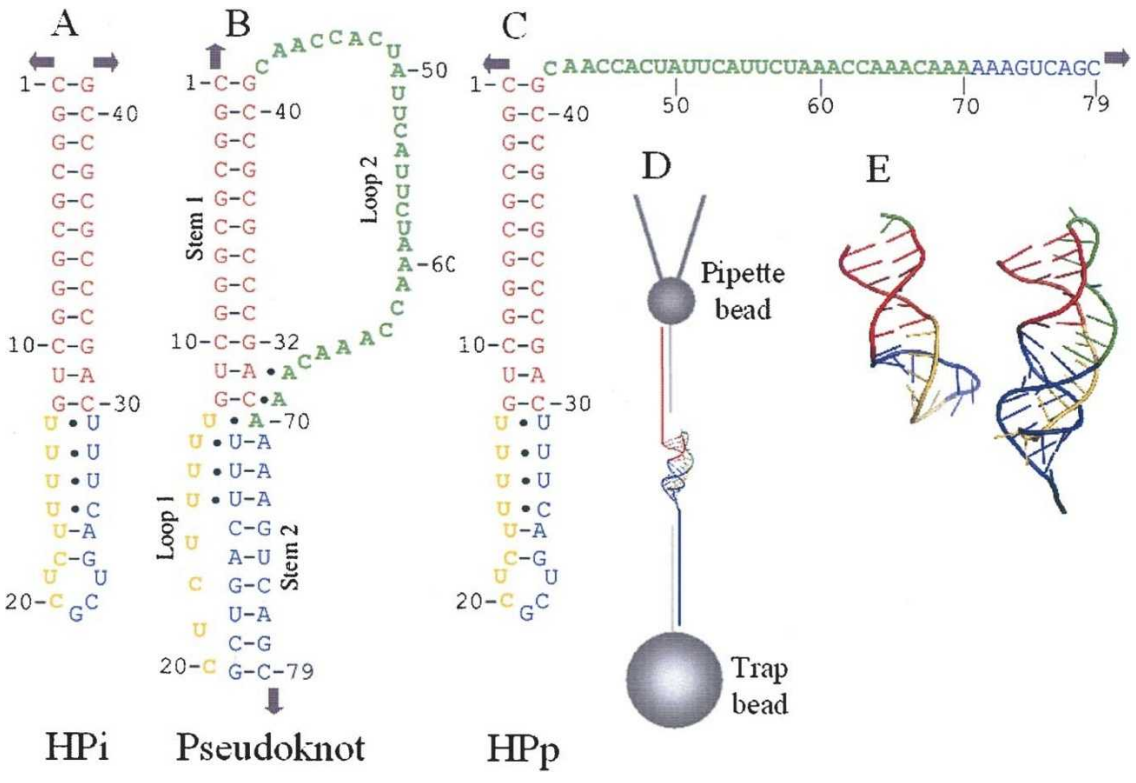

FIGURE 1. RNA constructs for single-molecule study. The constructs are derived from human telomerase RNA. The directions of stretching force by optical tweezers are shown by arrows. $(A)$ The isolated stem 1 hairpin construct (HPi). The noncanonical base pairs revealed by NMR are indicated with dots. The NMR construct of the stem 1 hairpin $(E$, left) consists of residues 7-35, with an additional $3^{\prime}$ dangling C. $(B)$ The pseudoknot construct. Tertiary contacts revealed by NMR are indicated with dots. The NMR construct of the pseudoknot $(E$, right) consists of the residues 7-35 and 63-79 (with residues 35 and 63 covalently linked), and an additional $3^{\prime}$ dangling A. The conserved single U-bulge (deleted in this construct) is located between residues 73 and 74. (C) The intermediate state during pseudoknot folding (HPp), in which the stem1 hairpin forms but stem2 does not. (D) The optical tweezers setup for single-molecule experiments. The RNA and DNA are shown in color and gray, respectively. The RNA molecule shown is from the NMR structure only for representation; only the structures shown in panels $A, B$, and $C$ were studied here. The drawing is not to scale. $(E)$ The three-dimensional NMR structures of the stem1 hairpin (Theimer et al. 2003) and pseudoknot derived from human telomerase RNA (Theimer et al. 2005).

2005), and because it is functionally essential to the catalytic activity of the telomerase ribonucleoprotein enzyme (Comolli et al. 2002; Chen and Greider 2005; Theimer et al. 2005).

To facilitate single-molecule mechanical unfolding and folding reactions, the pseudoknot was modified from the NMR construct (Theimer et al. 2005) and flanked by two DNA/RNA handles (with $\sim 500$ and $\sim 600$ base pairs [bp]), which are attached to two micron-sized polystyrene beads (Liphardt et al. 2001). One bead is held in a dual-beam force-measuring optical trap, and the other on a micropipette by suction (Fig. 1D). Force-jump and force-drop methods ( $\mathrm{Li}$ et al. 2006b) were employed to directly measure the unfolding and refolding kinetics. Stepwise force-drop experiments mapped the sequential folding of the pseudoknot structure and allowed direct measurement of the kinetics. Folding studies of the isolated $5^{\prime}$-hairpin construct (Fig. 1A) suggested that the $5^{\prime}$-hairpin within the pseudoknot (Fig. 1C) forms first, followed by formation of the $3^{\prime}$ stem. Transient formation of nonnative structures was observed with similar extension changes as native structures; these transient folding events reveal complex folding pathways for RNA.

\section{RESULTS}

\section{Constructs for single-molecule study}

The NMR structure of a truncated pseudoknot from human telomerase RNA (Fig. 1E, right panel) reveals extensive tertiary interactions (schematically shown in Fig. 1B) around the junction region involving sequences that are conserved among vertebrates (Theimer et al. 2005). The 5' (Fig. 1B, stem 1 , shown in red) and $3^{\prime}$ (Fig. 1B, stem2, shown in blue) stems of the pseudoknot are almost collinear. The NMR structure of an isolated stem 1 hairpin (Fig. 1E, left panel; Theimer et al. 2003) reveals that to form the phylogenetically conserved 9-bp A•Urich stem 2 and tertiary contacts of the pseudoknot, six base pairs (two canonical and four noncanonical) within the loop of stem1 hairpin (schematically shown in Fig. 1C) have to be broken (Theimer and Feigon 2006).

The modified human telomerase RNA pseudoknot construct (Fig. 1B) for single-molecule study is based on the NMR construct (Fig. 1E; Theimer et al. 2005), the wild-type pseudoknot, and functional analysis (Antal et al. 2002; Comolli et al. 2002; Chen and Greider 2004, 2005; Theimer et al. 2005). To follow the single-molecule reactions with distinct molecular extension changes $(>5 \mathrm{~nm})$ for individual reaction steps, stem1 and loop2 (Fig. 1B) were lengthened from the NMR construct. Stem 1 was lengthened from $6 \mathrm{bp}$ in the NMR construct to $12 \mathrm{bp}$, and the 29 nucleotides (nt) in loop2 were modified from the wild type. The loop1, which lies in the major groove of stem2, was maintained the same as in the NMR construct and the wild type. All the functionally important residues surrounding the junction (Comolli et al. 2002; Chen and Greider 2004, 2005; Theimer et al. 2005) were maintained except for the conserved single U-bulge in stem 2 between residues 73 and 74 (see Fig. 1B), which is also absent in the NMR study (Theimer et al. 2005). An isolated stem 1 hairpin construct (HPi) (Fig. 1A) was made by extending the DNA/RNA handle (see Materials and Methods) to cover the 3 '-side of the stem1 hairpin, thus preventing the formation of loop2 and stem2. The details of the NMR and single-molecule constructs are described in the Figure 1 caption. 


\section{Mechanical force range of pseudoknot unfolding/folding}

The force-ramp (with an approximately constant-force loading/unloading rate) method ( $\mathrm{Li}$ et al. 2006b) was first used to find the force ranges of individual unfolding/ folding reactions. In a force-ramp experiment (Fig. 2), the force on the RNA molecule is gradually increased or decreased; during the process, an unfolding/folding transition is indicated by a sudden increase/decrease in extension and decrease/increase in force. The abrupt unfolding and folding transitions are termed rips and zips, respectively. Representative force-ramp trajectories for the HPi construct are shown in Figure 2A. The HPi unfolding (Fig. 2A, gray traces) is indicated by a rip at $\sim 24$ piconewtons $(\mathrm{pN})$ with an extension increase of $\sim 18 \mathrm{~nm}$, consistent with the worm-like-chain model (Bustamante et al. 1994) for $41 \mathrm{nt}$
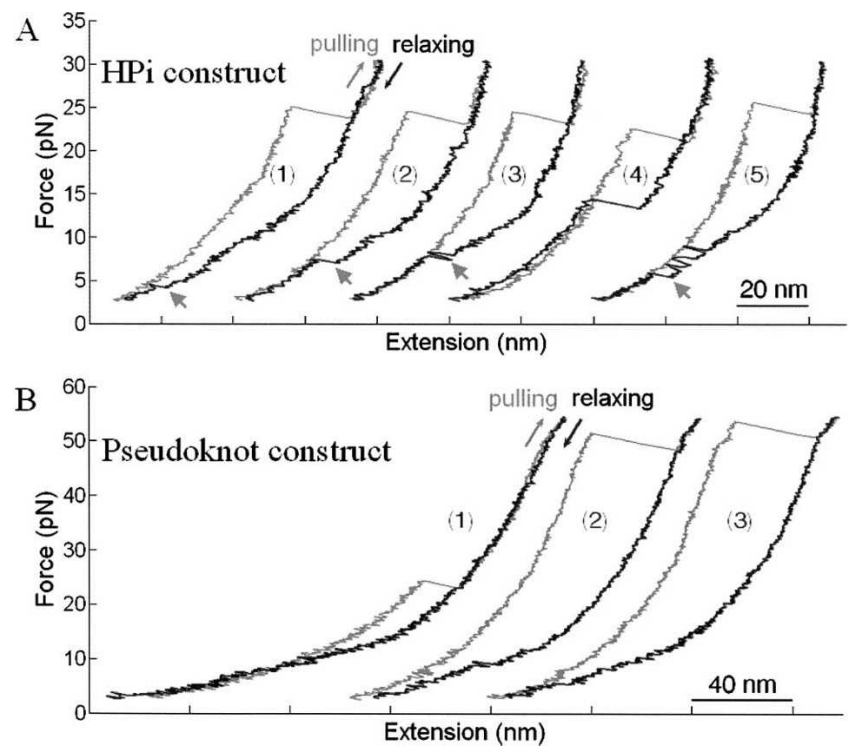

FIGURE 2. Representative force-extension curves from force-ramp experiments. The single RNA molecule is first pulled with a loading rate of $100 \mathrm{~nm} / \mathrm{sec}$ (shown in gray) and then relaxed with an unloading rate of $100 \mathrm{~nm} / \mathrm{sec}$ (shown in black). Stem 1 hairpin or pseudoknot unfolding transitions are indicated by the rips at $\sim 24$ and $\sim 50 \mathrm{pN}$, respectively. Folding zip transitions typically occur below $10 \mathrm{pN}$ and mostly are not resolved. (A) Five traces of force-ramp trajectories of the HPi construct, which can be characterized as three distinct types by the folding trajectories (see Results). Folding at or below $10 \mathrm{pN}$ with one folding zip: type (1), curves 1 and 2; and with multiple zipping/unzipping: type (2), curves 3 and 5. Curve 4 rarely occurs and belongs to type (3) with a folding zip above $10 \mathrm{pN}$. The folding zip transitions (indicated with gray arrows) shown in types (1) and (2) have a shorter extension change than that calculated by the worm-like-chain model for 41-nt single-stranded RNA. The multiple zipping/unzipping before the final folding zip transitions shown in type (2) might be due to formation/disruption of nonnative collapsed structures. The curves are ordered by occurrence from the highest to lowest. (B) Three traces of force-ramp trajectories of the pseudoknot construct. In curve 1 , only HPp forms as indicated by the unfolding force of $\sim 24 \mathrm{pN}$. In curves 2 and 3 , the pseudoknot forms as indicated by unfolding forces of $\sim 50 \mathrm{pN}$. of the hairpin unfolded, with a persistence length of $1 \mathrm{~nm}$ and contour length of $0.59 \mathrm{~nm} / \mathrm{nt}$ for single-stranded RNA (Table 1; Smith et al. 1996; Liphardt et al. 2001).

In contrast to unfolding, the folding of HPi occurred in a wide range of force. At least three types of folding processes (Fig. 2A, black traces) are observed for five molecules of $\mathrm{HPi}$, with a total of 193 traces: (1) Most (81\%) of the traces fold without a clear zip transition until below $10 \mathrm{pN}$, as shown in curves 1 and 2 ; (2) $\sim 16 \%$ of the traces show multiple zipping/unzipping processes at or below $10 \mathrm{pN}$ as shown in curves 3 and 5; and (3) only 3\% of the traces fold above 10 $\mathrm{pN}$ with a clear one-step zip as shown in curve 4 . Types (1) and (2), but not the rare events of type (3), were observed in all the molecules. The folding zip (Fig. 2A, indicated with gray arrows) at low forces $(\leq 10 \mathrm{pN})$ for the first two types have shorter extension change than that calculated by the worm-like-chain model for folding $41 \mathrm{nt}$ (Table 1); the difference is probably caused by the transient formation of small structures before the formation of HPi (see below).

In contrast to the hairpin structure, the pseudoknot structure unfolds in one step at relatively high force $(\sim 50$ $\mathrm{pN}$ ), with rip size of $\sim 36 \mathrm{~nm}$ (Fig. 2B, curves 2,3), consistent with the number of nucleotides expected for the unfolding of the pseudoknot (Table 1). The cooperative unfolding at high force of the pseudoknot is presumably due to the tertiary interactions, and to the fact that in pseudoknots the applied force is parallel to the helix axis of the stems (Fig. 1B, indicated with gray arrows). This applies a shear force to the stem, instead of an unzipping force that breaks base pairs one at a time, as in a hairpin (Fig. 1A,C, indicated with gray arrows). Unfolding a kissing hairpin complex is similar and also leads to unfolding forces higher than those for the component hairpins (Li et al. 2006a).

Distinct folding zip transitions are not consistently observed for the pseudoknot construct in its force-ramp relaxing trajectories (Fig. $2 \mathrm{~B}$ ). Some zips at $\sim 10 \mathrm{pN}$ (for example, Fig. 2B, curve 2) might be due to formation of the stem 1 hairpin within the pseudoknot construct (HPp) (Fig. 1C), as observed in the HPi construct (Fig. 2A). It is likely that the HPp folds into a pseudoknot below $10 \mathrm{pN}$, which is barely resolved in the force-ramp trajectory. The folding transition below $10 \mathrm{pN}$ is due to relatively slow folding rates compared to the force relaxation rate. Although the folding zip transitions are not resolved, the pseudoknot structure sometimes forms, as indicated by the subsequent pulling trajectories that show an unfolding force of $\sim 50 \mathrm{pN}$ and an extension increase of $\sim 36 \mathrm{~nm}$ (see Fig. $2 \mathrm{~B}$, curves $2,3)$. The pseudoknot construct sometimes shows similar unfolding force and extension changes as those of $\mathrm{HPi}$, indicating that only the HPp structure forms even when the force is decreased as low as $3 \mathrm{pN}$ (see Fig. 2B, curve 1). Thus, these results support the conclusion that HPp folds first at $\sim 10 \mathrm{pN}$ during the formation of the pseudoknot. Slow folding of HPp at or below $10 \mathrm{pN}$ masks the further folding zip transition to form the pseudoknot. 
TABLE 1. Parameters obtained from force-jump/drop experiments

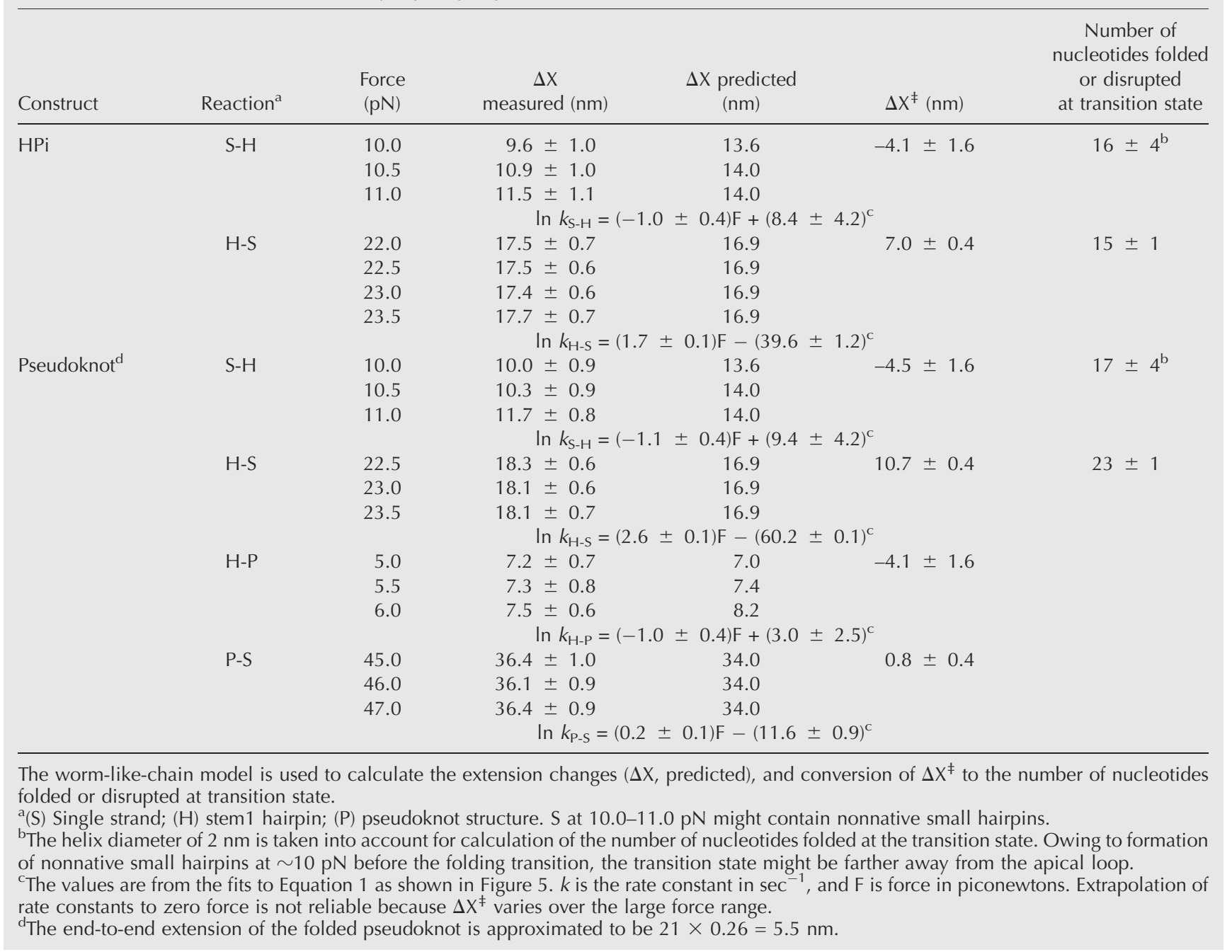

\section{Stem1 hairpin unfolding/folding kinetics}

As shown by force-ramp experiments (Fig. 2), formation of HPp and of pseudoknot structure is indicated by unfolding forces of $\sim 24$ and $\sim 50 \mathrm{pN}$, respectively. Both of the HPi and pseudoknot constructs fold at relatively low force (typically, $<10 \mathrm{pN}$ ), with folding zip transitions largely not resolved. To resolve the folding transitions, we employed force-jump and force-drop methods ( $\mathrm{Li}$ et al. 2006b). In a force-jump/drop experiment (Fig. 3), force is quickly $(<0.1 \mathrm{sec})$ increased/decreased to a preset force, and the preset force is kept constant by electronic force feedback. The unfolding/folding transition is indicated by a sudden increase/decrease of the molecular extension at constant force. The lifetimes of the molecule at each state (folded or unfolded) can be obtained for many cycles at different forces. By keeping the force above $9 \mathrm{pN}$, only HPp is observed to form; the pseudoknot structure does not form, thus allowing repetitive unfolding and folding of the
HPp within the pseudoknot construct. The HPp unfolds (H-S, transition from hairpin to single strand) at $\sim 23 \mathrm{pN}$ upon force-jump, with an abrupt molecular extension increase of $\sim 18 \mathrm{~nm}$, and folds ( $\mathrm{S}-\mathrm{H}$, transition from single strand to hairpin) at $\sim 10 \mathrm{pN}$ upon force-drop, with an abrupt extension decrease of $\sim 10 \mathrm{~nm}$ (Fig. 3; Table 1).

The folding transitions are $\sim 2-4 \mathrm{~nm}$ shorter in extension than those predicted by the worm-like-chain model for single-stranded RNA (Table 1), which might be due to formation of small hairpins before the folding transition at $\sim 10 \mathrm{pN}$. According to the nearest-neighbor model and a two-state mechanism for hairpin opening/closing (Tinoco et al. 1971; Serra et al. 1994; Xia et al. 1998; Mathews et al. 2004; Lu et al. 2006), the native 2-bp pentaloop between $\mathrm{U} 17$ and A25 (see Fig. 1C) has a predicted unfavorable free energy of $0.1 \mathrm{kcal} / \mathrm{mol}$ at zero force at $22^{\circ} \mathrm{C}$ in $1 \mathrm{M} \mathrm{NaCl}$. The melting temperature is predicted to be $21^{\circ} \mathrm{C}$ in $1 \mathrm{M}$ $\mathrm{NaCl}$. Thus, the $2-4-\mathrm{nm}$ deviation in extension is unlikely due to formation only of the native 2-bp pentaloop hairpin. 

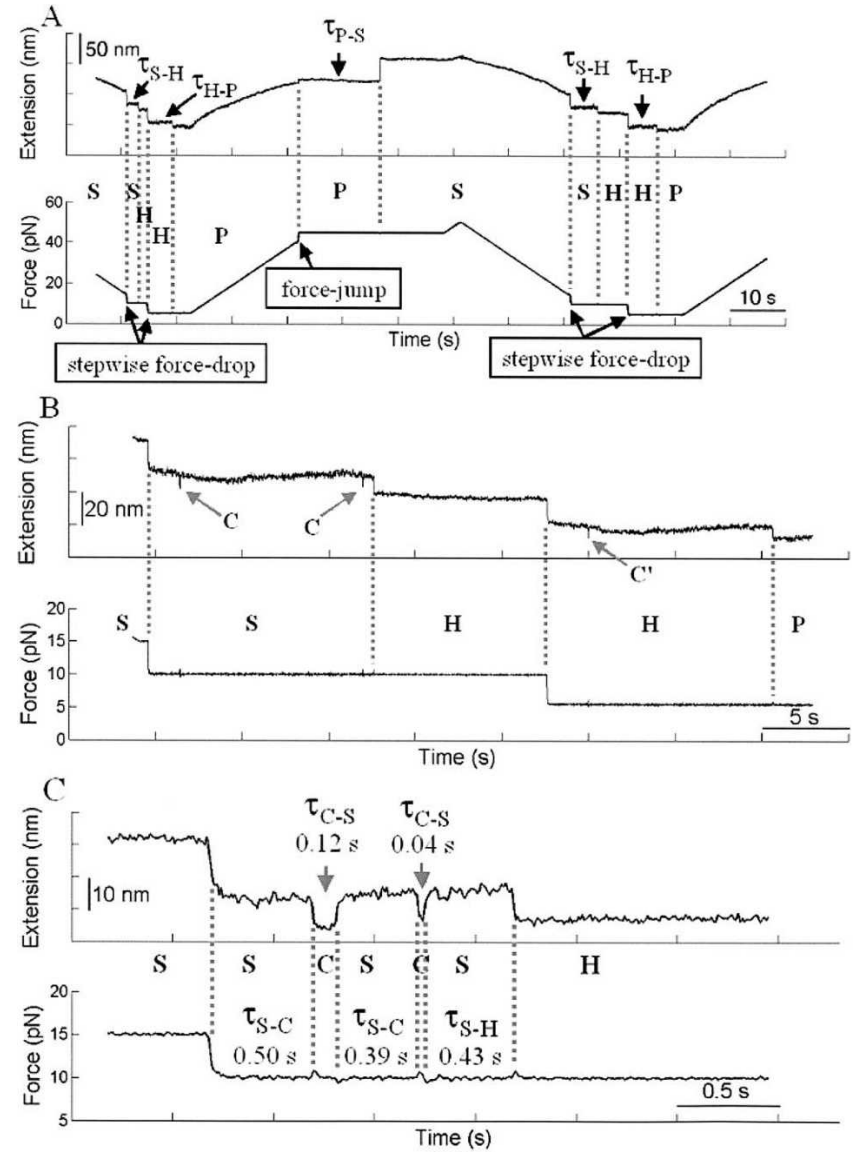

FIGURE 3. Representative force-jump/drop traces of the pseudoknot construct. Time courses of extension and force are shown on the top and bottom of each panel, respectively. Extension changes due to sudden force-jump/drop and unfolding/folding transition at constant force are indicated by vertical dashed lines. (S) Single strand; $(\mathrm{H})$ native stem1 hairpin; (P) native pseudoknot; (C) nonnative stem1 hairpin; $\left(\mathrm{C}^{\prime}\right)$ collapsed nonnative conformation of the pseudoknot. $\mathrm{S}$ at $\sim 10 \mathrm{pN}$ might contain nonnative small hairpins. (A) A trace without transient folding events. The stepwise force-drop (from 15.0 to $10.0 \mathrm{pN}$ and from 10.0 to $5.0 \mathrm{pN}$ ) and force-jump (from 40.0 to $45.0 \mathrm{pN})$ are indicated with black arrows. The lifetimes $(\tau)$ before unfolding/folding transitions are also shown. (B) A trace with two transient folding events (indicated with gray arrows) to nonnative HPp (S-C rapidly followed by C-S) before the formation of native stem1 hairpin $(\mathrm{S}-\mathrm{H})$ at $10.0 \mathrm{pN}$ and one transient folding event (indicated with gray arrows) to nonnative conformation of the pseudoknot construct ( $\mathrm{H}-\mathrm{C}^{\prime}$ rapidly followed by $\mathrm{C}^{\prime}-\mathrm{H}$ ) before the formation of the native pseudoknot (H-P) at $5.0 \mathrm{pN}$. (C) A close-up view of a trace with two transient folding events (indicated with gray arrows) to nonnative HPp (S-C followed by $\mathrm{C}-\mathrm{S}$ ). The measured lifetimes for single-stranded (S) and collapsed states (C) are shown.

It is probably due to formation of nonnative small hairpins. The nonnative small hairpins probably form during the force-drop process (during the dead time of the instrument, $<0.1 \mathrm{sec})$. Deviations from the expected extensions are also observed in the folding zip transitions below $10 \mathrm{pN}$ (Fig. 2A, indicated with gray arrows) in force-ramp trajectories. Presumably, the lifetime of the single-stranded RNA without any nonnative small hairpin at $\sim 10 \mathrm{pN}$ is too short to resolve, thus resulting in the apparent one-step native hairpin folding $(\mathrm{S}-\mathrm{H})$ transition with the ensemble kinetics listed in Table 2.

Repeated unfolding and folding of HPp within the pseudoknot by force-jump and force-drop, respectively, give single exponential distributions of lifetimes of the folded and unfolded molecules (Fig. 4); the respective ensemble kinetics parameters are $k_{\mathrm{H}-\mathrm{S}}=0.08-1.00 \mathrm{sec}^{-1}$ between 22.5 and $23.5 \mathrm{pN}$ and $k_{\mathrm{S}-\mathrm{H}}=0.07-0.21 \mathrm{sec}^{-1}$ between 10.0 and $11.0 \mathrm{pN}$ (Table 2). Thus, by force-drop experiments, the intermediate state with only HPp folded is available, and its folding kinetics can be directly measured. The unfolding and folding forces of $\sim 23$ and $\sim 10 \mathrm{pN}$ were chosen to provide experimentally accessible lifetimes.

The distances to transition states $\left(\Delta \mathrm{X}^{\ddagger}\right)$ along the reaction coordinate can be obtained from force-dependent unfolding/folding kinetics, $k(\mathrm{~F})$ (Fig. 5; Tables 1, 2; Tinoco et al. 2006):

$$
k(\mathrm{~F})=k_{0} \mathrm{e}^{\mathrm{F} \Delta \mathrm{X}^{\ddagger} / k_{B} T}
$$

where $k_{0}$ is the apparent unfolding/folding rate constant at zero force, $k_{\mathrm{B}}$ is the Boltzman constant, and $T$ is the temperature in kelvins. The value of $\Delta \mathrm{X}_{\mathrm{S}-\mathrm{H}}^{\ddagger}$ (from single strand to $\mathrm{HPp}$ ) is $-4.5 \pm 1.6 \mathrm{~nm}$ (Table 1), equivalent to the formation of a stem-loop containing $\sim 17 \mathrm{nt}$ (for example, a pentaloop with $\sim 6$ bp, i.e., from G12 to C30 in Fig. 1C). Due to likely formation of nonnative small hairpins, the single-stranded RNA deviates (with 2-4 nm shorter extension; see above) from the worm-like-chainmodel at $\sim 10 \mathrm{pN}$. Thus, the HPp folding transition state might be $2-4 \mathrm{~nm}(3-5 \mathrm{bp})$ further away from the apical loop. Possible nonnative local structures include the 5-bp triloop formed between G21 and C33 (Fig. 6; see Discussion). Note that the helix diameter of $2 \mathrm{~nm}$ has been taken into account $\left(-\Delta \mathrm{X}^{\ddagger}+2=4.5+2=6.5 \mathrm{~nm}\right)$ for calculation of the number of nucleotides folded at the transition state (Table 1). The NMR structure of an isolated stem1 hairpin (Fig. 1E, left panel; Theimer et al. 2003) reveals a single dominant conformation with four noncanonical base pairs (schematically shown in Fig. 1A). Presumably, the consecutive noncanonical $\mathrm{U} \bullet \mathrm{C}$ and $\mathrm{U} \bullet \mathrm{U}$ pairs observed in the NMR structure are not independently stable without closing Watson-Crick pairs on both sides (Chen and Turner 2006) at $\sim 10 \mathrm{pN}$. Thus, closing of the U-rich internal loop at $10.0-11.0 \mathrm{pN}$ is the rate-limiting step for the native stem 1 hairpin folding. Slow folding caused by the internal loop at the transition state measured here is consistent with slow folding caused by a 3-nt bulge observed previously in HIV TAR RNA (Li et al. 2006b).

The HPp unfolding transition state has $\Delta \mathrm{X}_{\mathrm{H}-\mathrm{S}}^{\ddagger}=10.7 \pm$ $0.4 \mathrm{~nm}$, corresponding to disruption of $\sim 23 \mathrm{nt}$ from the terminal ends, which is close to the U-rich internal loop (Fig. 1A; Table 1). It indicates that (nearly) all the 
TABLE 2. Ensemble reaction rate constants derived by single exponential fit $(k)$, and the reciprocal of average $(1 /\langle\tau\rangle)$ of the measured lifetimes from force-jump/drop experiments

\begin{tabular}{|c|c|c|c|c|c|c|c|}
\hline \multirow[b]{2}{*}{ Reaction $^{\mathrm{a}}$} & \multirow[b]{2}{*}{ Force $(\mathrm{pN})$} & \multicolumn{3}{|c|}{ Pseudoknot construct } & \multicolumn{3}{|c|}{ HPi construct } \\
\hline & & Number of traces & $k\left(\sec ^{-1}\right)$ & $1 /\langle\tau\rangle\left(\sec ^{-1}\right)$ & Number of traces & $k\left(\sec ^{-1}\right)$ & $1 /\langle\tau\rangle\left(\sec ^{-1}\right)$ \\
\hline \multirow[t]{3}{*}{$\mathrm{S}-\mathrm{H}$} & 10.0 & 132 & $0.21 \pm 0.01$ & 0.16 & 186 & $0.22 \pm 0.01$ & 0.17 \\
\hline & 10.5 & 121 & $0.17 \pm 0.01$ & 0.13 & 149 & $0.09 \pm 0.01$ & 0.08 \\
\hline & 11.0 & 125 & $0.07 \pm 0.01$ & 0.07 & 180 & $0.08 \pm 0.01$ & 0.07 \\
\hline \multirow[t]{3}{*}{ S-C } & 10.0 & 35 & $0.25 \pm 0.01$ & 0.25 & 53 & $0.36 \pm 0.01$ & 0.31 \\
\hline & 10.5 & 40 & $0.23 \pm 0.01$ & 0.21 & 11 & $0.15 \pm 0.02$ & 0.15 \\
\hline & 11.0 & 17 & $0.11 \pm 0.01$ & 0.12 & 9 & $0.09 \pm 0.01$ & 0.08 \\
\hline \multirow[t]{3}{*}{ C-S } & 10.0 & 35 & $21 \pm 1$ & 18 & 53 & $22 \pm 1$ & 17 \\
\hline & 10.5 & 40 & $35 \pm 2$ & 28 & 11 & $42 \pm 5$ & 19 \\
\hline & 11.0 & 17 & $30 \pm 4$ & 22 & 9 & $34 \pm 5$ & 30 \\
\hline \multirow[t]{4}{*}{$\mathrm{H}-\mathrm{S}$} & 22.0 & - & - & - & 136 & $0.06 \pm 0.01$ & 0.05 \\
\hline & 22.5 & 119 & $0.08 \pm 0.01$ & 0.08 & 154 & $0.12 \pm 0.01$ & 0.09 \\
\hline & 23.0 & 131 & $0.28 \pm 0.01$ & 0.27 & 165 & $0.28 \pm 0.01$ & 0.25 \\
\hline & 23.5 & 129 & $1.00 \pm 0.01$ & 0.87 & 77 & $0.67 \pm 0.01$ & 0.64 \\
\hline \multirow[t]{3}{*}{$\mathrm{H}-\mathrm{P}$} & 5.0 & 64 & $0.15 \pm 0.01^{b}$ & $0.12^{b}$ & & & \\
\hline & 5.5 & 91 & $0.13 \pm 0.01$ & 0.11 & & & \\
\hline & 6.0 & 89 & $0.06 \pm 0.01$ & 0.05 & & & \\
\hline \multirow[t]{3}{*}{$\mathrm{H}-\mathrm{C}^{\prime}$} & 5.0 & 17 & $0.27 \pm 0.02$ & 0.24 & & & \\
\hline & 5.5 & 24 & $0.14 \pm 0.01$ & 0.13 & & & \\
\hline & 6.0 & 26 & $0.10 \pm 0.01$ & 0.08 & & & \\
\hline \multirow[t]{4}{*}{$\mathrm{C}^{\prime}-\mathrm{H}$} & 5.0 & 17 & $21 \pm 2$ & 17 & & & \\
\hline & 5.5 & 24 & $2.3 \pm 0.1$ & 2.2 & & & \\
\hline & 6.0 & 26 & $12 \pm 1^{b}$ & $9^{b}$ & & & \\
\hline & 45.0 & 75 & $0.0359 \pm 0.0007$ & 0.0343 & & & \\
\hline \multirow[t]{2}{*}{ P-S } & 46.0 & 92 & $0.0417 \pm 0.0005$ & 0.0445 & & & \\
\hline & 47.0 & 111 & $0.0519 \pm 0.0009$ & 0.0510 & & & \\
\hline
\end{tabular}

Similar rate constants obtained by the two methods indicate apparent two-state reactions. Uncollapsing (C-S or $\left.\mathrm{C}^{\prime}-\mathrm{S}\right)$ rate constants are less accurate because of fewer traces obtained and short lifetimes of the collapsed states. Pseudoknot unfolding (P-S) rate constants are given extra significant digits for comparison.

${ }^{a}(\mathrm{~S})$ Single strand; $(\mathrm{H})$ native stem1 hairpin; $(\mathrm{P})$ native pseudoknot; $(\mathrm{C})$ nonnative stem1 hairpin; $\left(\mathrm{C}^{\prime}\right)$ collapsed nonnative conformation of the pseudoknot construct upon the second force-drop. $\mathrm{S}$ at $\sim 10 \mathrm{pN}$ might contain nonnative small hairpins.

${ }^{\mathrm{b}}$ One or two points are deleted with the longest lifetimes.

Watson-Crick pairs are disrupted at the unfolding transition state of HPp. The large block of $\mathrm{G} \bullet \mathrm{C}$-rich base pairs results in an unfolding force higher than $20 \mathrm{pN}$, a movement of the unfolding transition state closer to the apical hairpin loop, and hysteresis between folding and unfolding trajectories. These observations are consistent with previous modeling and experimental results (Liphardt et al. 2001; Vieregg and Tinoco 2006; Woodside et al. 2006a,b). Similar unfolding/folding kinetics data were obtained for the HPi construct (Fig. 5B; Tables 1, 2), as expected. In addition, folding of the 9-bp stem2 hairpin (with $41 \mathrm{nt}$ in the loop) before stem 1 is unlikely and would result in an end-to-end extension decrease of $\sim 20 \mathrm{~nm}$ at $\sim 10 \mathrm{pN}$, larger than we observed $(\sim 10 \mathrm{~nm})$. Therefore, we conclude that the stem1 hairpin folds (Fig. 1C) first and independently on the way to the formation of the pseudoknot structure (Fig. 1B). This folding pathway is consistent with that of thermal folding (Theimer et al. 2005).

\section{Pseudoknot unfolding (P-S) kinetics}

In contrast to the stepwise folding of the pseudoknot, onestep pseudoknot unfolding transitions ( $\mathrm{P}-\mathrm{S}$, transition from pseudoknot to single strand) are observed with force-jump experiments by rapidly increasing the force from 40 to $\sim 46$ pN (Fig. 3A). A sudden molecular extension increase of $\sim 36 \mathrm{~nm}$ with an ensemble rate constant of $0.05 \mathrm{sec}^{-1}$ is seen (Table 1,2), although our pseudoknot can sometimes unfold at forces lower than $40 \mathrm{pN}$ (with $\sim 5 \%$ frequency). The pseudoknot unfolding transition state at $\sim 46 \mathrm{pN}$ has $\Delta \mathrm{X}_{\mathrm{P}-\mathrm{S}}^{\ddagger}=0.8 \mathrm{~nm}$ (Table 1), which indicates that the unfolding transition state is close to the fully "pseudoknotted" native state at $46 \mathrm{pN}$, consistent with the brittle characteristics of tertiary interactions with unfolding kinetics relatively less force-dependent (Liphardt et al. 2001; Onoa et al. 2003). A small force range (45-47 pN) was used because higher forces result in breaking of the digoxigenin/ anti-digoxigenin antibody interactions (see Materials and 

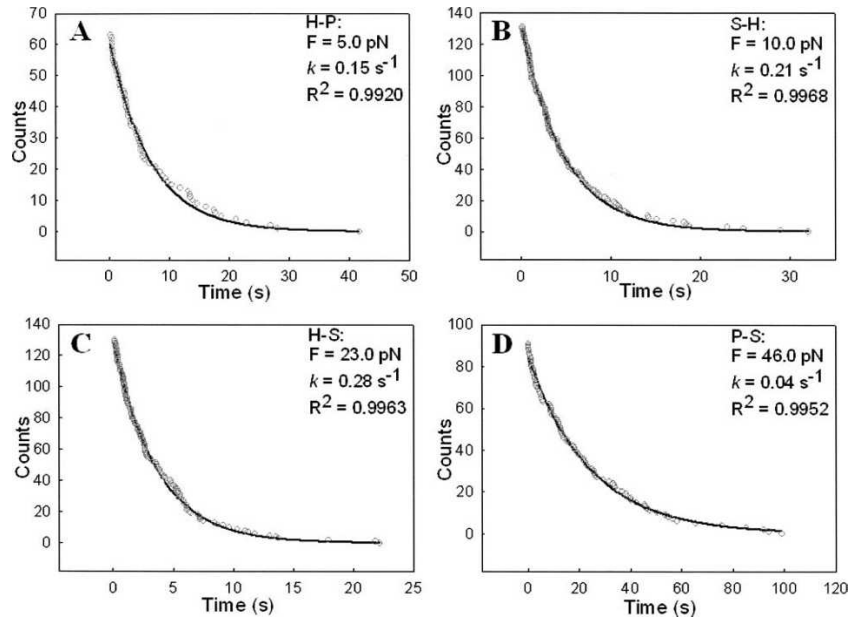

FIGURE 4. Representative plots of the counts of unreacted molecules as a function of time. Single exponential fits give the ensemble unfolding/folding rate constants at specific forces shown on top of each panel. (A) Pseudoknot folding from HPp (H-P) at $5.0 \mathrm{pN}$. (B) HPp folding from single strand $(\mathrm{S}-\mathrm{H})$ at $10.0 \mathrm{pN}$. (C) HPp unfolding to single strand $(\mathrm{H}-\mathrm{S})$ at $23.0 \mathrm{pN}$. (D) Pseudoknot unfolding to single strand $(\mathrm{P}-\mathrm{S})$ at $46.0 \mathrm{pN}$.

Methods), and lower forces result in an even slower unfolding rate.

\section{Pseudoknot folding (H-P) kinetics}

Once the HPp forms at $\sim 10 \mathrm{pN}$ after the first force-drop, the following pseudoknot folding transitions ( $\mathrm{H}-\mathrm{P}$, transition from hairpin to pseudoknot) are observed after a second force-drop by lowering the force rapidly from $\sim 10$ to $\sim 5 \mathrm{pN}$. A molecular extension decrease of $\sim 7 \mathrm{~nm}$ at $\sim 5$ $\mathrm{pN}$ occurs with an ensemble rate constant of $0.1 \mathrm{sec}^{-1}$ (Fig. 3; Table 2). The pseudoknot folding transition state has $\Delta \mathrm{X}_{\mathrm{H}-\mathrm{P}}^{\ddagger}=-4.1 \mathrm{~nm}$ (Table 1). To form the pseudoknot from HPp, the UCGCU pentaloop and six base pairs adjacent to the pentaloop formed between G12 and C30 (Fig. 1C), which are not significantly affected by the local force of $\sim 5$ $\mathrm{pN}$ exerted on terminal ends, have to be reorganized and (partially) opened to initiate successful base-pairing with the $3^{\prime}$-single-strand to form the 9-bp stem 2 of the pseudoknot (Fig. 1B). The $3^{\prime}$-single-strand is adenine-rich and designed to have no appreciable structures (Mathews et al. 2004). The value of $\Delta X_{\mathrm{H}-\mathrm{P}}^{\ddagger}=-4.1 \mathrm{~nm}$ is consistent with the fact that shortening of the extension of the 3 '-single-strand is required to base pair with the loop of HPp.

\section{Transient folding to nonnative structures of stem1 hairpin}

After force is quickly dropped to $\sim 10 \mathrm{pN}$, a stable native HPp structure forms with a sudden extension decrease, which is stable within our experimental time of up to 5 min. However, before HPp forms and after the force is stably maintained at the preset value, transient folding/ unfolding events are observed with folded lifetimes typically shorter than $0.1 \mathrm{sec}$, which are significantly shorter than that of the native stem 1 hairpin (Fig. 3B,C; Table 2). The corresponding extension changes $(\sim 10 \mathrm{~nm})$ are similar to those of native $\mathrm{HPp}$ folding transitions from single strand $(\mathrm{S}-\mathrm{H})$. We term this relatively short-lived state as a "collapsed state" (C), the transient folding transition as "collapsing" (S-C), and the unfolding transition from the collapsed state as "uncollapsing" (C-S). Transient folding to a collapsed state is also observed for the HPi construct (Table 2). There can be a mixture of collapsed structures with similar end-to-end extension changes as the native state. The native HPp unfolds with an ensemble rate constant of $0.08-1.00 \mathrm{sec}^{-1}$ between 22.5 and $23.5 \mathrm{pN}$ (Table 2). By extrapolation of the force-dependent kinetic equation: $\ln k_{\mathrm{H}-\mathrm{S}}=(2.6 \pm 0.1) \mathrm{F}-(60.2 \pm 0.1)$ (Fig. 5; Table 1), the native HPp would unfold with a calculated ensemble rate constant of near $10^{-14} \mathrm{sec}^{-1}$ at $10.0-11.0 \mathrm{pN}$, many orders of magnitude slower than the "uncollapsing" rate constant of $20-40 \mathrm{sec}^{-1}$ for the collapsed species at the same force (Table 2). Thus, the
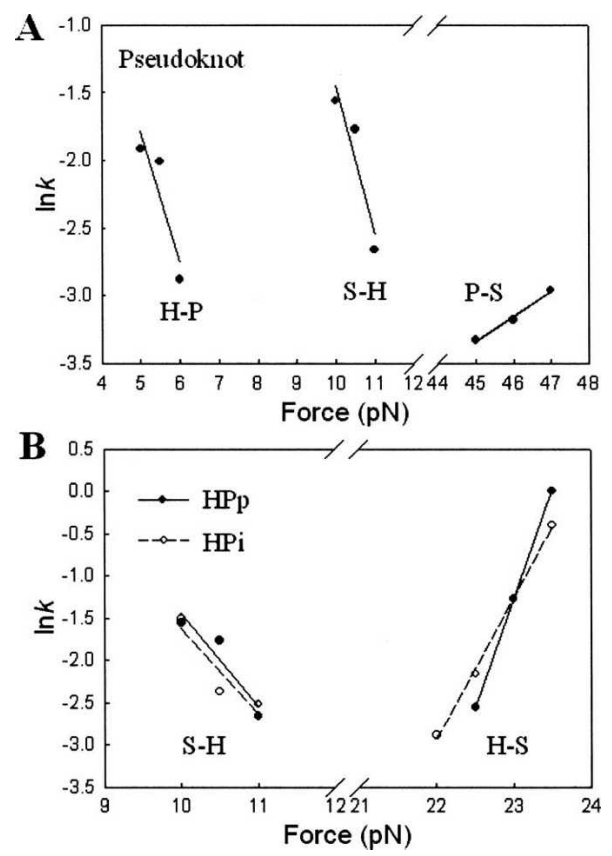

FIGURE 5. Plots of the natural logarithm of the ensemble rate constants $\left(\sec ^{-1}\right)$ versus force. The data are fit to Equation 1 with the fitting results shown in Table 1. (A) The pseudoknot construct. Pseudoknot folding $(\mathrm{H}-\mathrm{P})$ at $5.0-6.0 \mathrm{pN}$, HPp formation $(\mathrm{S}-\mathrm{H})$ at $10.0-11.0 \mathrm{pN}$, and pseudoknot unfolding (P-S) at 45.0-47.0 pN. (B) Steml hairpin unfolding (H-S) at $22.0-23.5 \mathrm{pN}$ and folding $(\mathrm{S}-\mathrm{H})$ at $10.0-11.0 \mathrm{pN}$ for $(\bigcirc)$ HPi construct and $(\bigcirc)$ HPp in the pseudoknot construct. Forcedependent ensemble reaction rate constants were measured covering a minimum force range of $1 \mathrm{pN}$ with $0.5-\mathrm{pN}$ intervals. Each data point on this figure was normally obtained from fitting of $>100$ transitions (see Fig. 4; Table 2). 


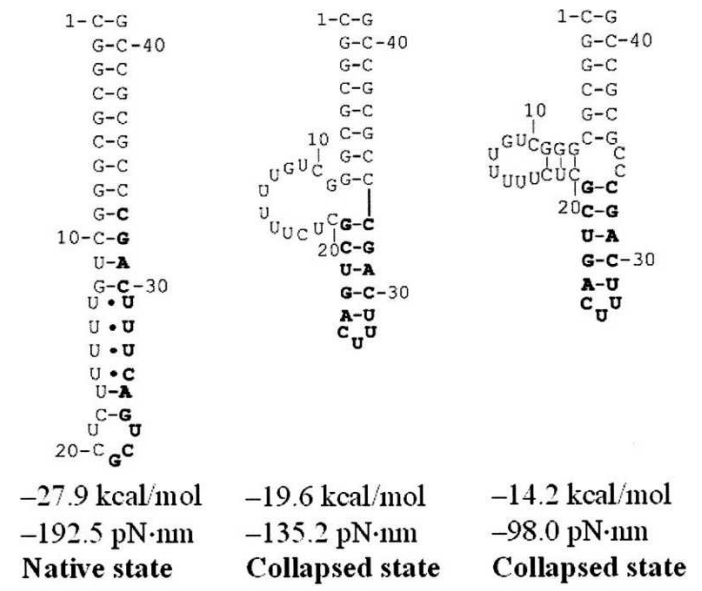

FIGURE 6. Native (left panel) and possible nonnative collapsed structures (middle and right panels) of the stem1 hairpin. The collapsed structures are predicted by the RNAstructure program. The free energies (in kilocalories per mole, at $37^{\circ} \mathrm{C}$ in $1 \mathrm{M} \mathrm{NaCl}$ ) and corresponding values in piconewtons-nanometers $(\mathrm{pN} \cdot \mathrm{nm})$ are shown at the bottom of each structure.

observed "collapsing/uncollapsing" transitions do not correspond to folding/unfolding of native HPp.

We observe one transient folding to nonnative HPp for approximately every seven constant-force trajectories at 11.0 $\mathrm{pN}$, and the frequency increases with lower force. The average collapsing rate constants $\left(k_{\mathrm{S}-\mathrm{C}}\right)$ differ by a factor of 2 or less between 10.0 and $11.0 \mathrm{pN}$, which is similar to the ratio of native folding rate constants $\left(k_{\mathrm{S}-\mathrm{H}}\right)$ (Table 2$)$. Note that, for those trajectories with transient folding events, the lifetime $\left(\tau_{\mathrm{S}-\mathrm{H}}\right.$, from the single-stranded conformation to native HPp or HPi structure) is calculated after the last uncollaping step, which is also done for the pseudoknot folding transition from native HPp ( $\tau_{\mathrm{H}-\mathrm{P}}$, see below) (Fig. $\left.3 \mathrm{C}\right)$.

To confirm that the transient folding is not due to the finite-speed electronic force feedback, we designed a different force-ramp experiment for the HPi construct. The molecule was pulled repeatedly between 10 and $30 \mathrm{pN}$, with $10 \mathrm{sec}$ of incubation time at $10 \mathrm{pN}$, in a passive mode, that is, without force feedback and thus without adjusting the pipette bead position (Fig. 7; Wen et al. 2007). As expected, the native HPi structure folds (S-H) at $\sim 10 \mathrm{pN}$ during the 10 -sec incubation period, as indicated by an abrupt force increase of $\sim 1 \mathrm{pN}$, corresponding to a molecular extension decrease $(\sim 10 \mathrm{~nm})$ upon folding. The formation of the native HPi conformation was confirmed by the subsequent force-ramp pulling trajectory with an unfolding force of $\sim 24 \mathrm{pN}$ (H-S) (see Fig. 7, top panel). Interestingly, transient force increase $(\sim 1 \mathrm{pN})$ events $(\mathrm{S}-\mathrm{C})$ were also observed in the passive mode trajectories before the formation of the native HPi structure, consistent with transient folding to nonnative collapsed structures (Fig. 7). Thus, occurrence of nonnative collapsed structures is not an artifact of the force-feedback mechanism.

\section{Transient folding to nonnative structures of the pseudoknot construct}

Upon the second force-drop by rapidly lowering force from $\sim 10$ to $\sim 5 \mathrm{pN}$, the pseudoknot folds from native $\mathrm{HPp}$ at $\sim 5 \mathrm{pN}$ with an ensemble rate constant of $0.1 \mathrm{sec}^{-1}$. Transient folding events are also observed in the trajectories of constant force with lifetimes typically shorter than 0.5 sec (Fig. 3B; Table 2). Analogous to the observation that transient folding events (collapsed states, C) occur before the formation of the native stem 1 hairpin (see above), these short-lived species are termed collapsed nonnative states of the pseudoknot construct $\left(\mathrm{C}^{\prime}\right)$. The native pseudoknot structure is stable once formed, as indicated by the absence of transient folding events.

Since the $3^{\prime}$-single-strand of steml (Fig. 1B,C) was designed to have no appreciable structure (Mathews et al. 2004), it is unlikely that the transient folding is due to local collapsing or folding from the $3^{\prime}$-single-strand. Thus, the collapsed nonnative conformations $\mathrm{C}^{\prime}$ are probably due to nonproductive intramolecular collisions, without enough stable interactions, between the loop of HPp and the $3^{\prime}$ single-strand. The frequency of collapsing events seems to be relatively independent of force from 5.0 to $6.0 \mathrm{pN}$, with similar collapsing rate constants as those of folding to native

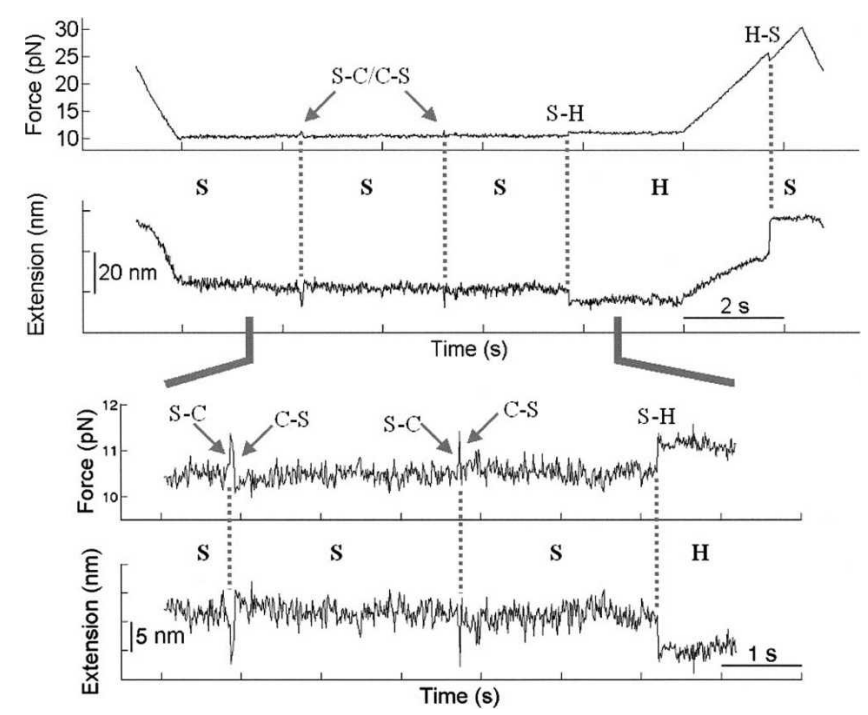

FIGURE 7. Transient folding ( $\mathrm{S}-\mathrm{C}$ rapidly followed by C-S, indicated with gray arrows) of the HPi construct during a passive mode experiment (without force feedback). The single strand (S) might contain nonnative small hairpins. Time courses of both force and extension are shown. Note that only the trap bead moves upon a transition during a passive mode and thus force increases and extension decreases for collapsing transition (S-C), but force decreases and extension increases rapidly for the following uncollapsing transition (C-S). Force increases and extension decreases when the native hairpin forms $(\mathrm{S}-\mathrm{H})$. Unfolding of the native stem 1 hairpin $(\mathrm{H}-$ $S$ ) is indicated by a rip at $\sim 24 \mathrm{pN}$ during the force-ramp. The bottom panel shows a close-up view of the top panel as indicated. For the force-ramp regions of this trace, the unloading rate is $200 \mathrm{~nm} / \mathrm{sec}$, and the loading rate is $100 \mathrm{~nm} / \mathrm{sec}$. 
pseudoknot $\left(k_{\mathrm{H}-\mathrm{P}}\right.$ versus $\left.k_{\mathrm{H}-\mathrm{C}^{\prime}}\right)$ (Table 2$)$. More folding trajectories with transient folding events are needed for a rigorous force-dependent collapsing and uncollapsing analysis.

\section{DISCUSSION}

Mechanical stretching forces perturb the RNA folding landscape by favoring the more extended conformations and facilitate direct mapping of unfolding/folding pathways and measurement of kinetics. Figure 8 shows the measured unfolding/folding kinetics of the dominant pathway of the modified human telomerase RNA pseudoknot in this study. The stochastic transient folding events suggest that RNA folding can take complex pathways and show multiple conformations (Figs. 3B,C, 7). Force can be used to distinguish the native and nonnative conformations with similar molecular extensions but different structural stability.

\section{Low-force folding and high-force unfolding}

The stem 1 hairpin and pseudoknot have different unfolding and folding forces and extension changes, which facilitate distinct observables for individual reactions (Fig. 8; Tables 1, 2). The force hysteresis larger than $10 \mathrm{pN}$ for stem 1 hairpin unfolding/folding (Fig. 2A) is mainly due to slow folding rates of the HPi and HPp, because of the ratelimiting step of closing the U-rich internal loop at the transition state (Fig. 1A,C). The folding forces of $10-11 \mathrm{pN}$ result in formation of nonnative small hairpins with extension decreases of 2-4 $\mathrm{nm}$. The lower limit for the disruption rate of one of the possible nonnative 5-bp

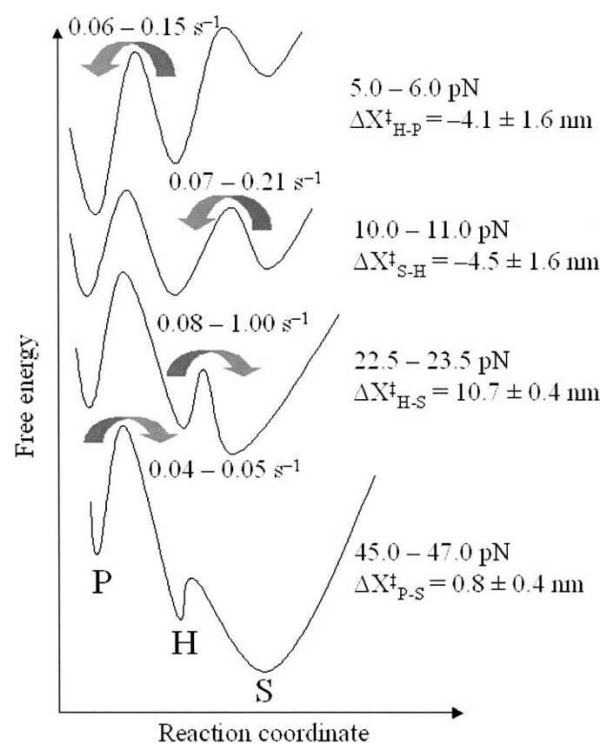

FIGURE 8. Schematic drawing of the folding landscapes involving the major conformational states of single-strand (S), stem1 hairpin $(\mathrm{H})$, and pseudoknot $(\mathrm{P})$ at different forces at $200 \mathrm{mM} \mathrm{NaCl}, 10 \mathrm{mM}$ Tris- $\mathrm{HCl}(\mathrm{pH} 7.3), 0.1 \mathrm{mM}$ EDTA, at room temperature $\left(22^{\circ} \mathrm{C}\right)$. Measured kinetic rate constants and distances to the transition states are also shown. triloops (formed between G21 and C33) (see Fig. 6) before the native folding transition is approximately measured to be $20-40 \mathrm{sec}^{-1}$ at zero force (see below). Thus, disruption of the nonnative small hairpins at $10.0-11.0 \mathrm{pN}$ is not the rate-limiting step for the native folding of the stem 1 hairpin $\left(k_{\text {S-H }}=0.07-0.21 \mathrm{sec}^{-1}\right)$.

Pseudoknot formation from HPp is even slower involving closing of the 29-nt loop2 (Fig. 1B,C), and it requires even lower force (Figs. 4, 5A). The mechanical stretching force is orthogonal to the stem 1 helix axis before the loop2 closing step (Fig. 1C, indicated with gray arrows), but the force becomes parallel to the helix axis after the pseudoknot forms (Fig. 1B, indicated with gray arrows). Requirement of low forces for folding was also observed for other structures, especially those involving tertiary interactions, such as a kissing hairpin complex (Li et al. 2006a) and the Tetrahymena group I ribozyme (Onoa et al. 2003).

In contrast to low-force folding, significantly higher force $(\sim 50 \mathrm{pN})$ is needed to unfold the pseudoknot. Relatively high force is required mainly because the force is applied parallel to the helix axis of the pseudoknot (a shearing mechanism) (see Fig. 1B). High-force unfolding by a shearing mechanism was observed previously for an RNA-kissing hairpin complex and for frameshift-inducing pseudoknots ( $\mathrm{Li}$ et al. 2006a; Green et al. 2007; Hansen et al. 2007). In contrast, relatively low force is needed to unfold a helix with force applied orthogonal to the helix axis (an unzipping mechanism) (see Fig. 1A,C). An unzipping mechanism breaks the secondary structure interactions one base pair at a time, and, thus, should have much lower energy barriers than a shearing mechanism.

Tertiary interactions, for example, compact consecutive base triples around the junction region add mechanical stability to the pseudoknot (Fig. 1B). These tertiary interactions form in the NMR construct (Fig. 1E; see Fig. $1 \mathrm{~B}$, base triples) in a buffer containing $200 \mathrm{mM} \mathrm{KCl}$ (Theimer et al. 2005). Thus, the tertiary interactions probably form in the pseudoknot construct used for the single-molecule study in $200 \mathrm{mM} \mathrm{NaCl}$ (Fig. 1B). Deletion of the single U-bulge (between residues 73 and 74) in stem2 (see the residue numbering in Fig. 1B) stabilizes the pseudoknot by $4 \mathrm{kcal} / \mathrm{mol}$ as measured for the NMR construct (Fig. 1E, right panel; Theimer et al. 2003, 2005), which affects unfolding pathways and adds mechanical stability. Magnesium ions stabilize the secondary and tertiary interactions of the pseudoknot further (Wyatt et al. 1990; Theimer and Giedroc 2000; Soto et al. 2007), which would require even higher unfolding forces $(>50 \mathrm{pN})$. The effect of magnesium ions was not studied here due to the mechanical stability limit of DNA/RNA handles, which can be overstretched above $60 \mathrm{pN}$ (Smith et al. 1996).

By ensemble thermal unfolding experiments in equilibrium, tertiary contacts were found to melt first followed by melting of stem 2 and finally melting of stem 1 for the NMR constructs (Fig. 1E; see Fig. 1B, structural scheme; 
Comolli et al. 2002; Theimer et al. 2003, 2005). With a shearing mechanism as studied here for the modified pseudoknot (Fig. 1B), mechanical unfolding disrupts tertiary and secondary structures cooperatively. Obviously, mechanical unfolding and thermal unfolding take different pathways. Force, as a vector, can unfold an RNA structure via different directions along the energy landscape and via nonequilibrium and equilibrium pathways with different kinetics. However, mechanical folding and thermal folding follow roughly the same pathway for the pseudoknot structure: first folding of stem 1 followed by stem 2 .

\section{Transient folding and possible collapsed structures}

Transient formations of nonnative collapsed structures ( $\mathrm{S}$ $\mathrm{C}$ and $\mathrm{H}-\mathrm{C}^{\prime}$ ) (Fig. 3B,C) are observed in both steps $(\mathrm{S}-\mathrm{H}$ and $\mathrm{H}-\mathrm{P}$ ) of the stepwise folding of the pseudoknot construct. The single strand $(\mathrm{S})$ at $\sim 10 \mathrm{pN}$ likely contains nonnative small hairpins (see below; Fig. 6). The collapsed nonnative states can be on-pathway or off-pathway to the native states. On-pathway collapsed states are directly converted to native state by thermal fluctuations with similar molecular extensions, and so they are not directly detectable in our experiments. The collapsed states we can detect are off-pathway.

Two possible nonnative structures of stem 1 hairpin predicted by the RNAstructure program (Mathews et al. 2004) are shown in Figure 6 (both containing the same nonnative 5-bp CUU triloop). Based on kinetic modeling of thermal folding (Cao and Chen 2007), an intermediate nonnative structure consisting of the 5-bp CUU triloop was also proposed for the 6-bp NMR construct (Fig. 1E; see Fig. 1A, sequence; Theimer et al. 2003). Perhaps the nonnative 5-bp CUU triloop structure (formed with the wild-type sequence between G21 and C33) has a faster folding rate than the native structure (formed between G12 and C30) (Fig. 6). Subsequent closing of the bulge or multibranch followed by formation of six to eight native $\mathrm{G} \bullet \mathrm{C}$ pairs adjacent to terminal ends (Fig. 6, middle and right panels) results in an apparent two-state transient folding with a similar molecular extension decrease as that of native stem 1 hairpin folding. As shown in Table 2, the force-dependent collapsing ( $\mathrm{S}-\mathrm{C}$ ) kinetics is similar to that of folding to the native hairpin $(\mathrm{S}-\mathrm{H})$. This proposed transient folding pathway follows the nucleation-zipping mechanism, that is, the nucleation of several closing base pairs is the rate-limiting step, which is followed by fast zipping of the remaining stem. It is less likely that the collapsing pathway is initiated by formation of several native $\mathrm{G} \bullet \mathrm{C}$ pairs adjacent to the terminal ends, which would have more force-dependent kinetics than we see here. It cannot be ruled out that multiple pathways are involved in the transient folding. More folding trajectories with transient folding events are needed for a rigorous force-dependent collapsing and uncollapsing analysis.
The postulated nonnative structures are significantly less stable than the native stem1 hairpin (Fig. 6). The work required to unfold the native stem 1 hairpin to form a single strand at $23 \mathrm{pN}$ is $414 \mathrm{pN} \cdot \mathrm{nm}(\mathrm{W}=\mathrm{F} \Delta \mathrm{X}$, including the stretching of single-stranded RNA of $\sim 100 \mathrm{pN} \cdot \mathrm{nm}$ ) (Tinoco 2004) with an extension increase of $18 \mathrm{~nm}$ (Table 1). In contrast, the work required to unfold the collapsed structure to nonnative small hairpins at $10 \mathrm{pN}$ is 100 $\mathrm{pN} \cdot \mathrm{nm}$ (including the stretching of single-stranded RNA of $\sim 35 \mathrm{pN} \cdot \mathrm{nm}$ ) (Tinoco 2004) with an extension increase of $10 \mathrm{~nm}$ (Fig. 3B,C), which is comparable to the predicted values at $37^{\circ} \mathrm{C}$ and $1 \mathrm{M} \mathrm{NaCl}$ at zero force (Fig. 6). Thus, the six or eight native $\mathrm{G} \bullet \mathrm{C}$ pairs in the proposed collapsed structures might be bistable at $\sim 10 \mathrm{pN}$ (considering the force, temperature, and salt effects), consistent with our observations.

When the nonnative collapsed structures of HPi or HPp form, on-pathway and off-pathway mechanisms may compete for the following steps. The six to eight native $\mathrm{G} \bullet \mathrm{C}$ pairs (Fig. 6, middle and right panels) under tension tend to unfold and result in an uncollapsing transition to singlestranded RNA (an off-pathway mechanism). However, the bottom part of the collapsed structures, including the nonnative 5-bp triloop favors the rearrangement to the more stable native hairpin conformation (see Fig. 6), resulting in an on-pathway reaction. Thus, the probability to follow the on- or off-pathway route to the hairpin formation is basically determined by the kinetics and stability of different parts of the collapsed structures. The force exerted on the terminal ends does not significantly affect the stability of the nonnative 5-bp triloop. Disruption of the nonnative 5-bp triloop is the rate-limiting step to reform the native stem 1 hairpin from the nonnative collapsed structures. Thus, the average uncollapsing rate (20-40 $\mathrm{sec}^{-1}$ ) (Table 2) provides a rough lower limit for the opening rate of the nonnative 5-bp triloop at zero force.

The disruption rate of the nonnative 5-bp triloop can also be predicted from equilibrium free energies assuming a hairpin formation rate $\left(k_{\text {formation }}\right)$ between $10^{4}$ and $10^{5} \mathrm{sec}^{-1}$ (Turner 2000). The free energy for formation of the nonnative 5-bp triloop without tension is predicted (Serra et al. 1994; Xia et al. 1998; Mathews et al. 2004; Lu et al. 2006) to be $-6.2 \mathrm{kcal} / \mathrm{mol}\left[K_{\mathrm{eq}}=\exp \left(-\Delta G_{\mathrm{i}} / R T\right)=4 \times\right.$ $\left.10^{4}\right]$ at $22^{\circ} \mathrm{C}$ at $1 \mathrm{M} \mathrm{NaCl}$. From the hairpin folding free energy and formation rate range at zero force, the hairpin disruption rate is calculated $\left(k_{\text {disrupton }}=k_{\text {formation }} / K_{\text {eq }}\right)$ to be $0.3-3 \mathrm{sec}^{-1}, \sim 1-2$ orders of magnitude slower than the uncollapsing rate observed (20-40 $\left.\mathrm{sec}^{-1}\right)$. Of course, fluctuation of the adjacent bulge or multibranch and lower salt concentration $(200 \mathrm{mM} \mathrm{NaCl})$ facilitate a faster opening rate than that predicted by only considering an isolated hairpin of the nonnative 5-bp triloop at $1 \mathrm{M} \mathrm{NaCl}$. Thus, the proposed collapsed structures are reasonable. It is also reasonable that native folding of the stem 1 hairpin can occur via rapid disruption of the nonnative 5-bp triloop at $\sim 10 \mathrm{pN}$. 
Transient folding during the second step of pseudoknot folding probably follows a "capture" mechanism, which was proposed for ribonucleoprotein assembly and RNA tertiary structure folding (Weeks and Cech 1996; Batey and Williamson 1998; Leulliot and Varani 2001; Chadalavada et al. 2002; Casiano-Negroni et al. 2007). It is probable that one of the pseudoknot folding transition pathways is initiated by reorganizing the UCGCU pentaloop formed between G12 and C30 (Fig. 1 C) and formation of several base pairs at the end of stem2 (Fig. 1B). Subsequent opening of the 6 bp (two Watson-Crick and four non-Watson-Crick) adjacent to the pentaloop, which is "captured" by the $3^{\prime}$ single-strand, leads to a successful pseudoknot folding transition (H-P). Accordingly, uncollapsing $\left(\mathrm{C}^{\prime}-\mathrm{H}\right)$ occurs if the adjacent $6 \mathrm{bp}$ are not opened for base-pairing with the $3^{\prime}$ single-strand. Such a pathway explains the observation of similar molecular extension decreases for native and transient pseudoknot folding. Other native and transient pseudoknot folding pathways are also possible (Cao and Chen 2007).

The force exerted on the terminal ends of stem 1 (Fig. 1C) does not significantly affect the opening rate of the pentaloop formed between G12 and C30 to initiate formation of stem 2 with the $3^{\prime}$-single-strand. Thus, the apparent forcedependent pseudoknot folding kinetics (Fig. 5A; Tables 1, 2) reflects the rate-limiting step of bringing the loop1 in proximity to the 3 '-single-strand and closing the 29-nt loop2 (Fig. 1B). The transition state positions for native and transient pseudoknot folding are less defined than that of hairpin folding. Our results provide a benchmark for testing theoretical simulations of the pseudoknot folding transition under tension in a more detailed molecular level, such as closing of loop 2 (Fig. 1B,C) by the $\sim 90^{\circ}$ flipping of stem 1 in HPp, by the reorganization of stem 1 hairpin loop residues (Theimer et al. 2003; Yingling and Shapiro 2005; Reipa et al. 2007), and by relaxation of the stretched 3 '-single-strand (Hyeon and Thirumalai 2006; Manosas et al. 2006).

\section{Transient folding characterized by force and molecular extension changes}

In the constant-force mode, the abrupt folding/unfolding transition with extension decrease/increase is accompanied by a small abrupt force increase/decrease (Wen et al. 2007). The force burst is due to the finite speed of force feedback, which is not fast enough to follow the trap bead movement when the RNA folds or unfolds, resulting in a time delay $(\sim 0.1 \mathrm{sec}$ in that system) before force is gradually restored to the preset value (Wen et al. 2007). Thus, the force burst amplitude is determined by the extent of extension changes upon folding/unfolding, and the effective stiffness of the optical trap and DNA/RNA handles. Such a force-feedback delay can cause a bistable RNA hairpin to fold/unfold at forces below/above preset values.

As expected, a force burst of up to $1 \mathrm{pN}$ is observed for both transient and native folding of the stem 1 hairpin at $\sim 10 \mathrm{pN}$ and is less significant for pseudoknot folding transition at $\sim 5 \mathrm{pN}$ (Fig. 3B,C). The restoring of the force to the preset values is significantly less than $0.1 \mathrm{sec}$ in the current system. Thus, lifetimes shorter than $0.1 \mathrm{sec}$ can still be accurately measured at constant force (see Fig. 3C; Manosas et al. 2007). Force bursts of up to $1 \mathrm{pN}$ from a preset value of $\sim 10 \mathrm{pN}$ or $\sim 5 \mathrm{pN}$ do not cause the native stem 1 hairpin or pseudoknot to unfold, because it is still far below the unfolding forces of the native stem 1 hairpin $(\sim 23 \mathrm{pN})$ and pseudoknot $(\sim 46 \mathrm{pN})$. In addition, transient folding is observed for the HPi construct in the passive mode at $\sim 10 \mathrm{pN}$ (without force feedback) (Fig. 7). Thus, the transient folding events we observed are not due to the finite time response of the force feedback, which, however, can affect the kinetics of uncollapsing (Table 2).

Transient sampling of other nonnative species (with extension changes smaller than $5 \mathrm{~nm}$ ) are also observed for HPi at $\sim 10 \mathrm{pN}$ and for the pseudoknot construct at $\sim 10$ and $\sim 5 \mathrm{pN}$ (data not shown). This is consistent with the fluctuations observed on the force-ramp relaxing trajectories below $10 \mathrm{pN}$, probably due to formation/disruption of local structures (Fig. 2; Li et al. 2007). These conformations, including the nonnative 5-bp triloop (Fig. 6), may result in smaller extension decreases on folding than that predicted by the worm-like-chain model at $10 \mathrm{pN}$ or below (Table 1; Smith et al. 1996; Liphardt et al. 2001). The loop2 and stem1 of the pseudoknot studied here were mutated to simplify the folding pathways, and thus we expect that more complex folding trajectories might be observed for the structure of the wild-type sequence (Gavory et al. 2006).

\section{Transient folding in other single-molecule and ensemble experiments}

Transient folding events of the pseudoknot construct are seen in both constant-force trajectories at $\sim 10$ and $\sim 5 \mathrm{pN}$ (Fig. 3B,C). The multiple zipping/unzipping events observed in force-ramp relaxing trajectories at $\sim 10 \mathrm{pN}$ or lower force might also be due to the transient formation of nonnative collapsed structures (see Fig. 2A, curves 3,5). Nonnative structures have been observed in both singlemolecule and ensemble experiments previously (Fang et al. 2002; Buchmueller and Weeks 2003; Tan et al. 2003; PerezSalas et al. 2004; Russell et al. 2006; Li et al. 2007). Misfolded structures were observed in HIV-1 TAR RNA folding by force-ramp and constant-force experiments by optical tweezers (Li et al. 2007). An intermediate state located at a single mismatch position was observed (lifetime $\sim 0.1 \mathrm{sec}$ ) for a DNA hairpin, with the fully folded state sampled transiently (lifetime $\sim 0.1-0.3 \mathrm{msec}$ ) (Woodside et al. 2006a). Multiple folding pathways for DNA and RNA hairpins were also suggested by ensemble temperature-jump studies (Ansari et al. 2001; Ma et al. 2006, 2007). Collapsed structures in protein mechanical folding have been observed by atomic force microscopy (Walther et al. 2007). 
FRET is a useful observable for detecting molecular extension changes for RNA tertiary structure unfolding/ folding ( $\mathrm{Ha}$ et al. 1999; Stone et al. 2007). Based on twostate analysis of single-molecule FRET trajectories, a collapsed state has been inferred as the transition state for intramolecular docking transitions between loops $\mathrm{A}$ and $\mathrm{B}$ of two-helix hairpin ribozymes (Bokinsky et al. 2003) and for GAAA tetraloop-receptor interactions (Hodak et al. 2005). Heterogeneity of the undocking kinetics of the hairpin ribozyme was attributed to formation of various near-native states with different stabilities (Bokinsky et al. 2003). A collapsed intermediate state was directly observed by single-molecule FRET in a four-way multibranch hairpin ribozyme with mutation of an essential $\mathrm{G} \bullet \mathrm{C}$ pair between loop A and loop B (Tan et al. 2003). A nearnative collapsed state was also observed for a cleaved form of the four-way multibranch hairpin ribozyme, presumably because of loss of native contact between "cleaved loop A" and loop B (Nahas et al. 2004).

Collapsed nonnative states have been observed by ensemble measurement for tertiary folding of the group I ribozyme and RNase P RNA (Fang et al. 2002; Buchmueller and Weeks 2003; Perez-Salas et al. 2004; Russell et al. 2006). Thus, near-native collapsed states can form in both secondary and tertiary structures. Studies from many functional RNA molecules indicate that misfolded or collapsed species can fold into native structures, probably facilitated by thermal fluctuation and/or mechanical force, in some ways similar to the action of chaperones.

By employing force-ramp and force-jump/drop methods, the short-lived nonnative collapsed states can be directly revealed, by following the complex folding pathways in real time. Utilization of both molecular extension and mechanical force provides powerful probes for perturbing and mapping the folding energy landscapes of both secondary and tertiary structures one molecule at a time. Compared with traditional methods to study RNA folding by varying solvent and temperature, the advantage of mechanical folding is that the experiments can be done under physiologically relevant conditions. Better understanding of RNA folding and unfolding may provide insight into therapeutics targeting telomerase.

\section{MATERIALS AND METHODS}

\section{Preparation of RNA and single-molecule constructs}

Single-molecule constructs were prepared as described previously (Liphardt et al. 2001). In brief, the chemically synthesized DNA oligonucleotide (Operon) corresponding to modified human telomerase RNA pseudoknot was cloned into the pBR322 vector (NEB) between the EcoRI and HindIII sites. The $\sim 1.2-\mathrm{kb}$ RNA with the pseudoknot sequence, flanked by $\sim 500$ and $\sim 600 \mathrm{nt}$, on the $5^{\prime}$ and $3^{\prime}$ halves, respectively, was in vitro transcribed. The corresponding $\sim 500$-bp (handle A) and $\sim 600$-bp (handle B) DNA sequences were generated by PCR. RNA was annealed with complementary strands of handle A and B by slowly cooling from $85^{\circ} \mathrm{C}$. A constituent hairpin construct (HPi) was made by extending the handle $\mathrm{B}$ to cover the $3^{\prime}$-side of the pseudoknot sequence. A biotin group was added to the $3^{\prime}$-end of handle A by biotin-linked dUTP (Roche) with T4 DNA polymerase (NEB); and a digoxigenin group was added at the $5^{\prime}$-end of the handle $\mathrm{B}$ via the primer (Operon) during PCR. The biotin and digoxigenin at the opposite ends of DNA/RNA handles provide affinity binding with streptavidin and anti-digoxigenin-coated polystyrene beads (Spherotech), respectively. During a single-molecule manipulation experiment, $\sim 2 \mu \mathrm{m}$ (diameter) streptavidin and $\sim 3$ $\mu \mathrm{m}$ anti-digoxigenin beads were held by micropipette and optical trap, respectively.

\section{Optical tweezers}

The force-measuring optical tweezers instrument described previously (830-nm laser) (Smith et al. 1996; Liphardt et al. 2001) was modified with a nanodrive piezoelectric stage (MCL), which can be moved and recorded with nanometer precision. Force was measured from the displacement of the trap bead. The extension change was measured from movement of the pipette bead, which was linked to the piezoelectric stage and the reaction chamber, and the trap bead.

\section{Force-ramp and force-jump/drop experiments}

The single-molecule mechanical unfolding/folding reactions were done at $200 \mathrm{mM} \mathrm{NaCl}, 10 \mathrm{mM}$ Tris- $\mathrm{HCl}$ (pH 7.3), $0.1 \mathrm{mM}$ EDTA, at room temperature $\left(22^{\circ} \mathrm{C} \pm 2^{\circ} \mathrm{C}\right)$. In a force-ramp experiment, the piezoelectric stage, which is linked to the micropipette bead, was moved back and forth continuously at a constant velocity (e.g., $100 \mathrm{~nm} / \mathrm{sec}$ ) (Smith et al. 1996; Liphardt et al. 2001; Li et al. 2006b). In a force-jump/drop experiment, an electronic feedback control was used to rapidly change and maintain the force ( $\mathrm{Li}$ et al. 2006b). A typical cycle of the force protocol is shown as follows:

(1) Unfolding: Force-ramp from 5 to $40 \mathrm{pN}$, followed by a fast force-jump to $46 \mathrm{pN}$, which is maintained constant until the pseudoknot unfolds as indicated by an abrupt extension increase.

(2) First force-drop: Force-ramp up to $50 \mathrm{pN}$ (to confirm complete structural unfolding) and then force-ramp down to $15 \mathrm{pN}$, followed by a force-drop to $10 \mathrm{pN}$ until stem 1 folds as indicated by abrupt extension decrease.

(3) Second force-drop: Force-drop to $5 \mathrm{pN}$ until pseudoknot folds as indicated by abrupt extension decrease.

The data were acquired at $100 \mathrm{~Hz}$ by a LabView program and analyzed by a MatLab program. The lifetimes obtained from the force-jump/drop experiment were sorted from shortest to longest. The ith lifetime $\left(\tau_{i}\right)$ was given a count of $(N-i)$, where $N$ is the total number of lifetimes obtained. The single exponential fits of $(N-i)$ versus $\tau_{i}$ give the ensemble rate constants for individual two-state reactions. The rate constants can also be obtained from the reciprocal of the average of all the lifetimes of individual reactions $(1 /\langle\tau\rangle)$. Similar rate constants obtained by the two methods indicate apparent two-state reactions. Uncollapsing rate constants are less accurate due to fewer data obtained and short lifetimes of the collapsed states. 


\section{ACKNOWLEDGMENTS}

We thank Mr. Jeffrey Vieregg for the instrument modification; Dr. Pan Li and Mr. Jeffrey Vieregg for critical reading of the manuscript; Dr. Steven Smith for some test experiments; Dr. Pan Li, Dr. Sophie Dumont, Dr. Lisa Green, Mr. Jeffrey Vieregg, and Dr. Steven Smith for help and discussions; and the Bustamante laboratory for sharing some instruments. This work was supported by NIH grant GM10840.

Received June 25, 2007; accepted September 9, 2007.

\section{REFERENCES}

Ansari, A., Kuznetsov, S.V., and Shen, Y. 2001. Configurational diffusion down a folding funnel describes the dynamics of DNA hairpins. Proc. Natl. Acad. Sci. 98: 7771-7776.

Antal, M., Boros, E., Solymosy, F., and Kiss, T. 2002. Analysis of the structure of human telomerase RNA in vivo. Nucleic Acids Res. 30: 912-920. doi: 10.1093/nar/30.4.912.

Autexier, C. and Lue, N.F. 2006. The structure and function of telomerase reverse transcriptase. Annu. Rev. Biochem. 75: 493-517.

Batey, R.T. and Williamson, J.R. 1998. Effects of polyvalent cations on the folding of an rRNA three-way junction and binding of ribosomal protein S15. RNA 4: 984-997.

Blackburn, E.H. 2006. Telomerase RNA. In The RNA world (eds. R.F. Gesteland et al.), pp. 419-436. Cold Spring Harbor Laboratory Press, Cold Spring Harbor, NY.

Bokinsky, G., Rueda, D., Misra, V.K., Rhodes, M.M., Gordus, A., Babcock, H.P., Walter, N.G., and Zhuang, X. 2003. Singlemolecule transition-state analysis of RNA folding. Proc. Natl. Acad. Sci. 100: 9302-9307.

Buchmueller, K.L. and Weeks, K.M. 2003. Near native structure in an RNA collapsed state. Biochemistry 42: 13869-13878.

Bustamante, C., Marko, J.F., Siggia, E.D., and Smith, S. 1994. Entropic elasticity of $\lambda$-phage DNA. Science 265: 1599-1600.

Bustamante, C., Chemla, Y.R., Forde, N.R., and Izhaky, D. 2004. Mechanical processes in biochemistry. Annu. Rev. Biochem. 73: 705-748.

Cao, S. and Chen, S.J. 2007. Biphasic folding kinetics of RNA pseudoknots and telomerase RNA activity. J. Mol. Biol. 367: 909-924.

Casiano-Negroni, A., Sun, X., and Al-Hashimi, H.M. 2007. Probing $\mathrm{Na}^{+}$-induced changes in the HIV-1 TAR conformational dynamics using NMR residual dipolar couplings: New insights into the role of counterions and electrostatic interactions in adaptive recognition. Biochemistry 46: 6525-6535.

Cech, T.R. 2004. Beginning to understand the end of the chromosome. Cell 116: 273-279.

Chadalavada, D.M., Senchak, S.E., and Bevilacqua, P.C. 2002. The folding pathway of the genomic hepatitis delta virus ribozyme is dominated by slow folding of the pseudoknots. J. Mol. Biol. 317: 559-575.

Chen, J.L. and Greider, C.W. 2004. Telomerase RNA structure and function: Implications for dyskeratosis congenita. Trends Biochem. Sci. 29: 183-192.

Chen, J.L. and Greider, C.W. 2005. Functional analysis of the pseudoknot structure in human telomerase RNA. Proc. Natl. Acad. Sci. 102: 8080-8085.

Chen, G. and Turner, D.H. 2006. Consecutive GA pairs stabilize medium-size RNA internal loops. Biochemistry 45: 4025-4043.

Collins, K. 2006. The biogenesis and regulation of telomerase holoenzymes. Nat. Rev. Mol. Cell Biol. 7: 484-494.

Comolli, L.R., Smirnov, I., Xu, L., Blackburn, E.H., and James, T.L. 2002. A molecular switch underlies a human telomerase disease. Proc. Natl. Acad. Sci. 99: 16998-17003.

Fang, X.W., Thiyagarajan, P., Sosnick, T.R., and Pan, T. 2002. The rate-limiting step in the folding of a large ribozyme without kinetic traps. Proc. Natl. Acad. Sci. 99: 8518-8523.
Gavory, G., Symmons, M.F., Krishnan Ghosh, Y., Klenerman, D., and Balasubramanian, S. 2006. Structural analysis of the catalytic core of human telomerase RNA by FRET and molecular modeling. Biochemistry 45: 13304-13311.

Gesteland, R.F., Cech, T.R., and Atkins, J.F., eds. 2006. The RNA world. Cold Spring Harbor Laboratory Press, Cold Spring Harbor, NY.

Green, L., Kim, C.H., Bustamante, C., and Tinoco Jr., I. 2007. Characterization of the mechanical unfolding of RNA pseudoknots. J. Mol. Biol. (in press). doi: 10.1016/j.jmb.2007.05.058.

Ha, T., Zhuang, X., Kim, H.D., Orr, J.W., Williamson, J.R., and Chu, S. 1999. Ligand-induced conformational changes observed in single RNA molecules. Proc. Natl. Acad. Sci. 96: 9077-9082.

Hansen, T.M., Reihani, S.N., Oddershede, L.B., and Sorensen, M.A. 2007. Correlation between mechanical strength of messenger RNA pseudoknots and ribosomal frameshifting. Proc. Natl. Acad. Sci. 104: 5830-5835.

Hodak, J.H., Downey, C.D., Fiore, J.L., Pardi, A., and Nesbitt, D.J. 2005. Docking kinetics and equilibrium of a GAAA tetraloopreceptor motif probed by single-molecule FRET. Proc. Natl. Acad. Sci. 102: 10505-10510.

Hyeon, C. and Thirumalai, D. 2006. Forced-unfolding and forcequench refolding of RNA hairpins. Biophys. J. 90: 3410-3427.

Legassie, J.D. and Jarstfer, M.B. 2006. The unmasking of telomerase. Structure 14: 1603-1609.

Leontis, N.B., Lescoute, A., and Westhof, E. 2006. The building blocks and motifs of RNA architecture. Curr. Opin. Struct. Biol. 16: 279287.

Leulliot, N. and Varani, G. 2001. Current topics in RNA-protein recognition: Control of specificity and biological function through induced fit and conformational capture. Biochemistry 40: 79477956.

Li, P.T.X., Bustamante, C., and Tinoco Jr., I. 2006a. Unusual mechanical stability of a minimal RNA kissing complex. Proc. Natl. Acad. Sci. 103: 15847-15852.

Li, P.T.X., Collin, D., Smith, S.B., Bustamante, C., and Tinoco Jr., I. 2006b. Probing the mechanical folding kinetics of TAR RNA by hopping, force-jump, and force-ramp methods. Biophys. J. 90: 250-260.

Li, P.T.X., Bustamante, C., and Tinoco Jr., I. 2007. Real-time control of the energy landscape by force directs the folding of RNA molecules. Proc. Natl. Acad. Sci. 104: 7039-7044.

Liphardt, J., Onoa, B., Smith, S.B., Tinoco Jr., I., and Bustamante, C. 2001. Reversible unfolding of single RNA molecules by mechanical force. Science 292: 733-737.

Lu, Z.J., Turner, D.H., and Mathews, D.H. 2006. A set of nearest neighbor parameters for predicting the enthalpy change of RNA secondary structure formation. Nucleic Acids Res. 34: 4912-4924. doi: 10.1093/nar/gkl472.

Ma, H., Proctor, D.J., Kierzek, E., Kierzek, R., Bevilacqua, P.C., and Gruebele, M. 2006. Exploring the energy landscape of a small RNA hairpin. J. Am. Chem. Soc. 128: 1523-1530.

Ma, H., Wan, C., Wu, A., and Zewail, A.H. 2007. DNA folding and melting observed in real time redefine the energy landscape. Proc. Natl. Acad. Sci. 104: 712-716.

Manosas, M., Collin, D., and Ritort, F. 2006. Force-dependent fragility in RNA hairpins. Phys. Rev. Lett. 96: 218-301.

Manosas, M., Wen, J.D., Li, P.T.X., Smith, S.B., Bustamante, C., Tinoco Jr., I., and Ritort, F. 2007. Force unfolding kinetics of RNA using optical tweezers. II. Modeling experiments. Biophys. J. 92: 3010-3021.

Mathews, D.H., Disney, M.D., Childs, J.L., Schroeder, S.J., Zuker, M., and Turner, D.H. 2004. Incorporating chemical modification constraints into a dynamic programming algorithm for prediction of RNA secondary structure. Proc. Natl. Acad. Sci. 101: 7287-7292.

Nahas, M.K., Wilson, T.J., Hohng, S., Jarvie, K., Lilley, D.M.J., and Ha, T. 2004. Observation of internal cleavage and ligation reactions of a ribozyme. Nat. Struct. Mol. Biol. 11: 1107-1113.

Onoa, B., Dumont, S., Liphardt, J., Smith, S.B., Tinoco Jr., I., and Bustamante, C. 2003. Identifying kinetic barriers to mechanical unfolding of the T. thermophila ribozyme. Science 299: 1892-1895. 
Perez-Salas, U.A., Rangan, P., Krueger, S., Briber, R.M., Thirumalai, D., and Woodson, S.A. 2004. Compaction of a bacterial group I ribozyme coincides with the assembly of core helices. Biochemistry 43: 1746-1753.

Pleij, C.W., Rietveld, K., and Bosch, L. 1985. A new principle of RNA folding based on pseudoknotting. Nucleic Acids Res. 13: 17171731. doi: 10.1093/nar/13.5.1717.

Puglisi, J.D., Wyatt, J.R., and Tinoco Jr., I. 1988. A pseudoknotted RNA oligonucleotide. Nature 331: 283-286.

Reipa, V., Niaura, G., and Atha, D.H. 2007. Conformational analysis of the telomerase RNA pseudoknot hairpin by Raman spectroscopy. RNA 13: 108-115.

Russell, R., Das, R., Suh, H., Travers, K.J., Laederach, A., Engelhardt, M.A., and Herschlag, D. 2006. The paradoxical behavior of a highly structured misfolded intermediate in RNA folding. J. Mol. Biol. 363: 531-544.

Serra, M.J., Axenson, T.J., and Turner, D.H. 1994. A model for the stabilities of RNA hairpins based on a study of the sequence dependence of stability for hairpins of six nucleotides. Biochemistry 33: $14289-14296$.

Smith, S.B., Cui, Y., and Bustamante, C. 1996. Overstretching B-DNA: The elastic response of individual double-stranded and singlestranded DNA molecules. Science 271: 795-799.

Soto, A.M., Misra, V., and Draper, D.E. 2007. Tertiary structure of an RNA pseudoknot is stabilized by "diffuse" $\mathrm{Mg}^{2+}$ ions. Biochemistry 46: 2973-2983.

Staple, D.W. and Butcher, S.E. 2005. Pseudoknots: RNA structures with diverse functions. PLoS Biol. 3: e213. doi: 10.1371/journal. pbio. 0030213 .

Stone, M.D., Mihalusova, M., O’Connor, C.M., Prathapam, R., Collins, K., and Zhuang, X. 2007. Stepwise protein-mediated RNA folding directs assembly of telomerase ribonucleoprotein. Nature 446: 458-461.

Su, L., Chen, L., Egli, M., Berger, J.M., and Rich, A. 1999. Minor groove RNA triplex in the crystal structure of a ribosomal frameshifting viral pseudoknot. Nat. Struct. Biol. 6: 285-292.

Tan, E., Wilson, T.J., Nahas, M.K., Clegg, R.M., Lilley, D.M.J., and Ha, T. 2003. A four-way junction accelerates hairpin ribozyme folding via a discrete intermediate. Proc. Natl. Acad. Sci. 100: 9308-9313.

Theimer, C.A. and Feigon, J. 2006. Structure and function of telomerase RNA. Curr. Opin. Struct. Biol. 16: 307-318.

Theimer, C.A. and Giedroc, D.P. 2000. Contribution of the intercalated adenosine at the helical junction to the stability of the gagpro frameshifting pseudoknot from mouse mammary tumor virus. RNA 6: 409-421.

Theimer, C.A., Finger, L.D., Trantirek, L., and Feigon, J. 2003. Mutations linked to dyskeratosis congenita cause changes in the structural equilibrium in telomerase RNA. Proc. Natl. Acad. Sci. 100: 449-454.
Theimer, C.A., Blois, C.A., and Feigon, J. 2005. Structure of the human telomerase RNA pseudoknot reveals conserved tertiary interactions essential for function. Mol. Cell 17: 671-682.

Tinoco Jr., I. 1996. Nucleic acid structures, energetics, and dynamics. J. Phys. Chem. 100: 13311-13322.

Tinoco Jr., I. 2004. Force as a useful variable in reactions: Unfolding RNA. Annu. Rev. Biophys. Biomol. Struct. 33: 363-385.

Tinoco Jr., I. and Bustamante, C. 1999. How RNA folds. J. Mol. Biol. 293: $271-281$.

Tinoco Jr., I., Uhlenbeck, O.C., and Levine, M.D. 1971. Estimation of secondary structure in ribonucleic acids. Nature 230: 362-367.

Tinoco Jr., I., Li, P.T.X., and Bustamante, C. 2006. Determination of thermodynamics and kinetics of RNA reactions by force. Q. Rev. Biophys. 39: 325-360.

Turner, D.H. 2000. Conformational changes. In Nucleic acids: Structures, properties, and functions (eds. V.A. Bloomfield et al.), pp. 259-334. University Science Books, Sausalito, CA.

Vieregg, J.R. and Tinoco Jr., I. 2006. Modeling RNA folding under mechanical tension. Mol. Phys. 104: 1343-1352.

Walther, K.A., Grater, F., Dougan, L., Badilla, C.L., Berne, B.J., and Fernandez, J.M. 2007. Signatures of hydrophobic collapse in extended proteins captured with force spectroscopy. Proc. Natl. Acad. Sci. 104: 7916-7921.

Weeks, K.M. and Cech, T.R. 1996. Assembly of a ribonucleoprotein catalyst by tertiary structure capture. Science 271: 345-348.

Wen, J.D., Manosas, M., Li, P.T.X., Smith, S.B., Bustamante, C., Ritort, F., and Tinoco Jr., I. 2007. Force unfolding kinetics of RNA using optical tweezers. I. Effects of experimental variables on measured results. Biophys. J. 92: 2996-3009.

Woodside, M.T., Anthony, P.C., Behnke-Parks, W.M., Larizadeh, K., Herschlag, D., and Block, S.M. 2006a. Direct measurement of the full, sequence-dependent folding landscape of a nucleic acid. Science 314: 1001-1004.

Woodside, M.T., Behnke-Parks, W.M., Larizadeh, K., Travers, K., Herschlag, D., and Block, S.M. 2006b. Nanomechanical measurements of the sequence-dependent folding landscapes of single nucleic acid hairpins. Proc. Natl. Acad. Sci. 103: 61906195.

Wyatt, J.R., Puglisi, J.D., and Tinoco Jr., I. 1990. RNA pseudoknots: Stability and loop size requirements. J. Mol. Biol. 214: 455-470.

Xia, T., SantaLucia Jr., J., Burkard, M.E., Kierzek, R., Schroeder, S.J., Jiao, X., Cox, C., and Turner, D.H. 1998. Thermodynamic parameters for an expanded nearest-neighbor model for formation of RNA duplexes with Watson-Crick base pairs. Biochemistry 37: 14719-14735.

Yingling, Y.G. and Shapiro, B.A. 2005. Dynamic behavior of the telomerase RNA hairpin structure and its relationship to dyskeratosis congenita. J. Mol. Biol. 348: 27-42. 

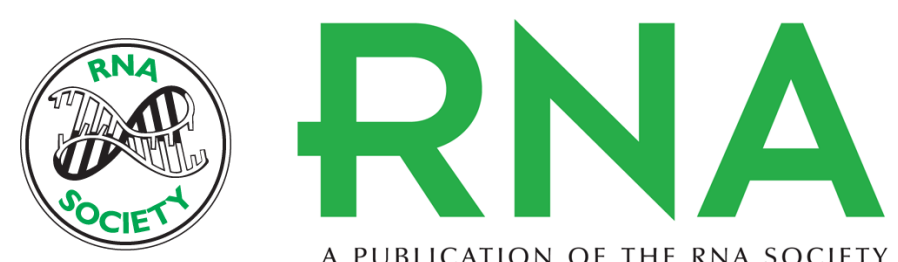

A PUBLICATION OF THE RNA SOCIETY

\section{Single-molecule mechanical unfolding and folding of a pseudoknot in human telomerase RNA}

Gang Chen, Jin-Der Wen and Ignacio Tinoco, Jr

RNA 2007 13: 2175-2188

References This article cites 68 articles, 25 of which can be accessed free at:

http://rnajournal.cshlp.org/content/13/12/2175.full.html\#ref-list-1

License

Email Alerting Receive free email alerts when new articles cite this article - sign up in the box at the Service top right corner of the article or click here. 